\title{
Dimer and String Formation during Low Temperature Silicon Deposition on Si(100)
}

\author{
Smith, A. P.; Jonsson, Hannes
}

Published in:

Physical Review Letters

Link to article, DOI:

10.1103/PhysRevLett.77.1326

Publication date:

1996

Document Version

Publisher's PDF, also known as Version of record

Link back to DTU Orbit

Citation $(A P A)$ :

Smith, A. P., \& Jonsson, H. (1996). Dimer and String Formation during Low Temperature Silicon Deposition on Si(100). Physical Review Letters, 77(7), 1326-1329. https://doi.org/10.1103/PhysRevLett.77.1326

\section{General rights}

Copyright and moral rights for the publications made accessible in the public portal are retained by the authors and/or other copyright owners and it is a condition of accessing publications that users recognise and abide by the legal requirements associated with these rights.

- Users may download and print one copy of any publication from the public portal for the purpose of private study or research.

- You may not further distribute the material or use it for any profit-making activity or commercial gain

- You may freely distribute the URL identifying the publication in the public portal

If you believe that this document breaches copyright please contact us providing details, and we will remove access to the work immediately and investigate your claim. 


\title{
Dimer and String Formation during Low Temperature Silicon Deposition on Si(100)
}

\author{
A.P. Smith ${ }^{1}$ and H. Jónsson ${ }^{1,2}$ \\ ${ }^{1}$ Department of Chemistry, University of Washington, Box 351700, Seattle, Washington 98195-1700 \\ ${ }^{2}$ Center for Atomic-Scale Materials Physics, Department of Physics, Technical University of Denmark, DK-2800 Lyngby, Denmark
}

(Received 13 March 1996)

\begin{abstract}
We present theoretical results based on density functional theory and kinetic Monte Carlo simulations of silicon deposition and address observations made in recently reported low temperature scanning tunneling microscopy studies. A mechanism is presented which explains dimer formation on top of the substrate's dimer rows at $160 \mathrm{~K}$ and up to room temperature, while between-row dimers and longer strings of adatoms ("diluted dimer rows") form at higher temperature. A crossover occurs at around room temperature between two different mechanisms for adatom diffusion in our model. [S0031-9007(96)00849-6]
\end{abstract}

PACS numbers: 68.35.Fx, 68.35.Bs, 81.15.Hi

Advanced materials technology often involves growing high-quality metastable structures. For systems far from thermodynamic equilibrium, a successful growth scheme giving the desired morphology maintains some control over competing dynamical processes. Thin film growth by deposition of atoms onto a surface is an important technique, and the key dynamical processes are diffusion of adatoms on the surface and the formation of dimers, trimers, and larger clusters resulting in nucleation of new islands. Much of the research on film growth by deposition has focused on silicon, which has become the canonical example for studies of covalent crystal growth [1].

A great deal of effort has been devoted to studies of the growth dynamics of the $\mathrm{Si}(100)$ surface. Brocks, Kelly, and Car [2] used electron density functional theory (DFT) and the local density approximation (LDA) to predict a nonepitaxial (i.e., different from bulk crystal) binding site for the adatom. This site is to the side of the dimer rows formed by the $(2 \times 1)$ reconstruction of the $\mathrm{Si}(100)$ surface (the $S$ sites shown in Fig. 1). Diffusion occurs through hops between neighboring optimal sites, with an estimated activation energy barrier of $0.6 \mathrm{eV}$ along the direction of the dimer rows and $1.0 \mathrm{eV}$ for perpendicular diffusion. This picture for single atom diffusion has been generally confirmed in experiments over a range of temperatures: Adatoms have been observed at the predicted binding site in scanning tunneling microscopy (STM) images [3] at $160 \mathrm{~K}$, and their immobility implies an activation energy of at least $0.56 \mathrm{eV}$. An upper bound of $0.67 \mathrm{eV}$ for the activation energy for diffusion parallel to dimer rows and an estimate of $1.0 \mathrm{eV}$ for diffusion perpendicular to the rows was extracted by Mo et al. [4] from analysis of island size distribution and "denuded zones" at step edges observed in STM images.

While these basic features of the adatom binding and dynamics are now well established, several puzzling observations have been made in recent, low temperature STM measurements of addimers and larger clusters. The accuracy of DFT calculations has, in particular, been questioned [5]. We present here a mechanism for adatom dynamics and cluster formation which is consistent with the main features of the STM measurements and is founded on energetics obtained from DFT calculations using the gradient dependent PW91 functional [6]. While the results obtained using the PW91 functional are qualitatively similar to the LDA results, the quantitative differences in diffusion barriers are large enough to significantly change the deduced atomic scale dynamics in the relevant temperature range. We first present our DFT results on energetics and then discuss the dynamics at various temperatures.

The DFT results were obtained by calculations similar to those described previously [7,8]. They make use of a Car-Parrinello [9] type algorithm to optimize both electronic degrees of freedom and atomic arrangement for a surface consisting of one to three adatoms on a slab of 8 layers with $8 \mathrm{Si}$ atoms per layer. As before, a 12 Ry energy cutoff and the $\Gamma$ point were used in these calculations, and Ref. [7] discusses the reliability of the approach in some detail: Relative energies are estimated

\section{Si (100)2x1 adatom sites}

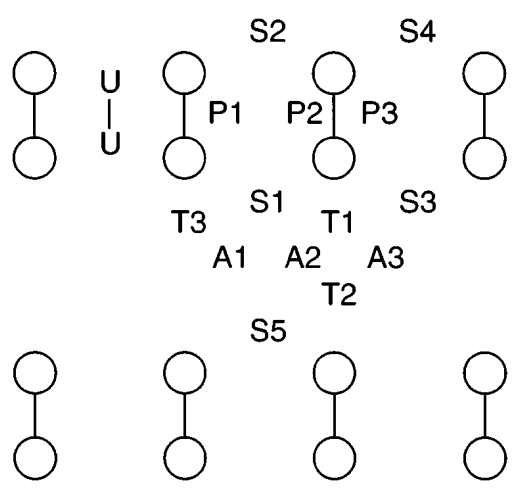

FIG. 1. Segments of two dimer rows of the $(2 \times 1)$ reconstructed $\mathrm{Si}(100)$ surface are shown and the four types of stable adatom sites $S, T, P$, and $A$. The numbering of the different equivalent sites is the basis for labeling various stable addimer configurations referred to in Table II. The $U-U$ dimer configuration, which does not directly correspond to stable adatom sites, is also shown. 
to be converged to roughly $0.1 \mathrm{eV}$, but the LDA functional can give relative energies that differ from those obtained by the gradient dependent PW91 functional by up to $0.4 \mathrm{eV}$. The energies and diffusion hop barriers for the four stable binding sites ( $S, P, T$, and $A$, see Fig. 1), obtained previously [7], are summarized in Table I, and new results on the energies of various dimer and trimer structures are given in Table II. The addimer configurations were generated by placing the two adatoms near stable sites for individual adatoms, while the substrate atoms had the configuration of a clean, staggered $p(2 \times 2)$ surface. The configurations were then relaxed to a local minimum in the total energy. Three separate DFT-LDA calculations have previously been reported for some of these configurations [2,10,11]. There is quite good agreement among the different DFT-LDA calculations (see Table II). All the DFT numbers (including our DFT-PW91 results) show that the lowest energy is obtained when the addimer is placed on top of the dimer rows (the $U-U$ configuration, shown in Fig. 1, is less than $0.1 \mathrm{eV}$ higher in energy than $P 1-P 2$ within DFT-PW91). The $S 1-S 5$ addimer consisting of atoms in two neighboring $S$ sites is higher in energy, and, surprisingly, has the same energy as the $S 1 \cdots S 2$ configuration, where the two adatoms are not directly bonded to each other. The three atom string, $S 2 \cdots S 1-S 5$, is very stable, with bond energies comparable to those of the individual dimers. The DFT results, therefore, indicate the observed [12], longer " $S$ strings" are quite stable.

Table I gives the expected time between adatom hops over the various diffusion barriers $E^{a}$ based on the approximation $\tau_{\text {hop }}=(\nu)^{-1} \exp \left(\beta E^{a}\right)$ and assuming a prefactor $\nu=10^{13} \mathrm{sec}^{-1}$, typical for diffusion processes. For a given time scale, new dynamical processes become active as the substrate temperature is raised. Calculated and observed barriers to dimer motion (except for rotation between the two on-top configurations) are roughly $1 \mathrm{eV}$ or higher [11,13], meaning the dimers are immobile at room temperature and the abundance of observed addimer configurations indicates the relative importance of different kinetic pathways for their formation.

The starting point for these kinetic pathways is the initial site occupied by a newly deposited atom. Theoretical

TABLE I. Activation energy barriers for diffusion hops obtained from the DFT-PW91 calculation. The hopping time $\tau$ $(\mathrm{sec})$ is estimated for the three temperatures discussed in the text. The energies of the $P, T$, and $A$ sites above the $S$ site are $0.22,0.59$, and $0.86 \mathrm{eV}$, respectively.

\begin{tabular}{lclll}
\hline \hline \multicolumn{1}{c}{ Hop } & Barrier $(\mathrm{eV})$ & $\tau(160 \mathrm{~K})$ & $\tau(300 \mathrm{~K})$ & $\tau(400 \mathrm{~K})$ \\
\hline$E_{P 2 \rightarrow P 3}^{a}$ & 0.27 & $3 \times 10^{-5}$ & $3 \times 10^{-9}$ & \\
$E_{P 1 \rightarrow P 2}^{a}$ & 0.34 & $5 \times 10^{-3}$ & $5 \times 10^{-8}$ & \\
$E_{P \rightarrow S}^{a}$ & 0.49 & $3 \times 10^{2}$ & $2 \times 10^{-5}$ & $1 \times 10^{-7}$ \\
$E_{S \rightarrow T}^{a}$ & 0.60 & & $1 \times 10^{-3}$ & $4 \times 10^{-6}$ \\
$E_{S \rightarrow P}^{a}$ & 0.71 & & $8 \times 10^{-2}$ & $1 \times 10^{-4}$ \\
$E_{S \rightarrow A}^{a}$ & 0.91 & & $2 \times 10^{2}$ & $3 \times 10^{-2}$ \\
\hline \hline
\end{tabular}

modeling of homoepitaxial growth [14] suggests rapid thermalization of newly deposited atoms [15] before they have time to move any significant distance on the surface. We can approximate the capture cross section for the four stable binding sites by the area of the attractive wells in the DFT-PW91 calculated potential energy surface. Atoms landing near the $A$ and $T$ sites will quickly find the nearby $S$ site and get trapped there, since the barriers for those hops are only $0.1 \mathrm{eV}$. But atoms landing in a $P$ site have to overcome a large barrier of $0.5 \mathrm{eV}$ to get to an $S$ site, while the barrier to hopping along the top of the dimer row, from one $P$ site to another, is only about $0.3 \mathrm{eV}$. Therefore adatoms landing on top of rows (roughly $45 \%$ according to Fig. 6 of Ref. [7]) will be able to travel some distance along this channel before visiting one of the $S$ sites. Note that this differs from DFT-LDA calculations [16], for which the $P$ site adatoms would not move far before landing in a neighboring $S$ site. A similar, low barrier mechanism for diffusion along dimer rows has also been suggested by Lu, Zhang, and Metiu [17] based on calculations using the empirical Stillinger-Weber potential $[18,19]$.

The DFT-PW91 energy surface and the predicted thermally activated hopping dynamics (see Table I) are quite consistent with the experimental measurements of Wolkow [3] at $160 \mathrm{~K}$. The STM images showed mainly immobile and isolated adatoms at $S$ sites, but about $40 \%$ of the deposited atoms were found as addimers sitting on top of dimer rows [3], which could only be explained by some

TABLE II. Energy (in eV) of dimer and trimer configurations relative to adatoms at $S$ sites, also compared with recent results from YUT [11] and BK [10] (BK do not quote energies relative to adatoms, so the $P 1-P 2$ number is assumed the same as reported by YUT). In parentheses is the energy difference from the corresponding isolated adatoms (leftmost column), indicating bond strength. The two adatoms are close enough to appear bonded in the first nine configurations. The $U-U$ configuration shown in Fig. 1 is nearly degenerate with $P 1-P 2$.

\begin{tabular}{lrrrr}
\hline \hline & YUT & BK & LDA & \multicolumn{1}{c}{ PW91 } \\
\hline$P_{1}-P_{2}$ & -0.76 & -0.76 & -0.7 & $-0.9(-1.3)$ \\
$S 1-S 5$ & -0.58 & -0.45 & -0.5 & $-0.4(-0.4)$ \\
$A 1-A 2$ & 0.00 & 0.35 & -0.1 & $0.1(-1.6)$ \\
$S 1-A 1$ & & & 0.3 & $0.5(-0.4)$ \\
$P 2-P 3$ & & & 0.3 & $0.5(0.0)$ \\
$P 2-T 1$ & & & 0.2 & $0.6(-0.2)$ \\
$T 1-A 2$ & & & 0.6 & $0.6(-0.8)$ \\
$A 2-A 3$ & & & 1.1 & $1.1(-0.6)$ \\
$T 1-T 2$ & & & 0.9 & $1.1(-0.1)$ \\
& -0.56 & & -0.3 & $-0.4(-0.4)$ \\
$S 1 \cdots S 2$ & & & -0.1 & $-0.2(-0.2)$ \\
$S 1 \cdots S 4$ & & & 0.2 & $0.1(-0.2)$ \\
$P 1 \cdots S 5$ & & & 0.3 & $0.3(0.3)$ \\
$S 1 \cdots S 3$ & & & 0.3 & $0.4(0.1)$ \\
$S 1 \cdots P 3$ & & & 1.0 & $0.6(-0.1)$ \\
$P 1 \cdots A 2$ & & & 0.7 & $0.8(0.2)$ \\
$S 1 \cdots T 2$ & & & & \\
$S 2 \cdots S 1-S 5$ & -0.99 & -1.01 & -1.0 & $-1.0(-1.0)$ \\
\hline \hline
\end{tabular}


form of "transient mobility" of the adatoms. The potential channel described above indeed leads to transient mobility as metastable on-top adatoms travel long distances by rapid, thermally activated hopping. Eventually, they run into one another and form $P-P$ dimers, with some fraction lost to hops into the immobile $S$ sites. On-top addimers may also form when a diffusing atom passes close to an immobile $S$ adatom. In our calculations, where we start with a $P 1-S 1$ adatom pair, the structural relaxation results in the formation of a $U-U$ dimer.

Several experiments on silicon deposition close to room temperature have been carried out $[5,12,13,20]$. Addimers on top of dimer rows are still a dominant feature at low coverage (the $P 1-P 2$ and $U-U$ orientations of the on-top dimer are now in equilibrium $[5,12]$ ), but additional structures are also observed, and the isolated adatoms have disappeared. Mo et al. [20] found that deposition between 300 and $650 \mathrm{~K}$ produced "diluted dimer rows," strings of adatoms on the surface with only half the adatom density of regular $\mathrm{Si}(100)$ dimer rows. Bedrossian's high resolution STM images, showed the features consist of $S$ strings, rows of adatoms sitting near the optimal $S$ binding sites (as in $S 2 \cdots S 1-S 5$ in Fig. 1) [12]. Zhang et al. [5] reported about a third of the addimers sitting in the troughs in between dimer rows (in particular, $S 1-S 5$ dimers) rather than on top.

The kinetic pathways for addimer and cluster formation are more complex near room temperature, but they must still derive from the motion of single adatoms. We have carried out kinetic Monte Carlo (kMC) simulations of the deposition process [21]. Each run consisted of 20 atoms deposited over $1-10 \mathrm{sec}$ in a $6 \times 800$ surface cell, with equilibration continuing for another $10 \mathrm{sec}$, giving a deposition rate and coverage analogous to the laboratory experiments. Adatoms are allowed to hop between $S$ and $P$ sites, and they interact according to the dimer interactions derived from those sites, using energies and barriers from Tables I and II. The $U-U$ dimers are not included explicitly except through the zero-barrier formation of on-top dimers from adatoms at $P 1-$ and $S 1$ type sites.

Our initial kMC simulations used the rates given in Table I, with the result that essentially all the deposited adatoms ended up in dimers on top of the dimer rows (of $P 1-P 2$ type, which we from now on denote $P-P$ ) at room temperature and above. This happens to be the most stable dimer configuration, but the reason for its dominance here is that diffusion along the top of the dimer rows is many times more effective than diffusion along the edges, even though the overall activation barrier is $0.1 \mathrm{eV}$ lower for the latter mechanism. The two mechanisms can be composed by realizing that the $S$ and $P$ sites are locally in equilibrium on the time scale of these experiments. The fraction of the time spent on top is then given by the energy difference between the $S$ and $P$ sites, $\exp \left(-\beta \Delta E_{S, P}\right)$, neglecting entropic effects. The frequency of hops along the row once the adatom is on top is $\nu_{P \rightarrow P} \exp \left(-\beta E_{P \rightarrow P}^{a}\right)$, so the overall rate of hops along the top of a row is

$$
k_{\text {top }}=\nu_{P \rightarrow P} \exp \left[-\beta\left(\Delta E_{S, P}+E_{P \rightarrow P}^{a}\right)\right] .
$$

The frequency of hops along the edge (via $T$ sites) is

$$
k_{\text {edge }}=\nu_{S \rightarrow S} \exp \left(-\beta E_{S \rightarrow S}^{a}\right) .
$$

Assuming the prefactors $\nu_{P \rightarrow P}$ and $\nu_{S \rightarrow S}$ are the same, ontop diffusion will dominate if $E_{S \rightarrow S}^{a}>\Delta E_{S, P}+E_{P \rightarrow P}^{a}$ even though the hopping barrier is larger, $E_{S \rightarrow P}^{a}>E_{S \rightarrow S}^{a}$. Using our DFT-PW91 binding energies and barriers, this condition holds by $0.04 \mathrm{eV}$.

This explains the propensity for $P-P$ dimerization in the $\mathrm{kMC}$ simulations, and also suggests how the rates can be modified to better reproduce the experiments of Zhang et al. and Bedrossian [5,12], where a substantial fraction of the adatoms were found as dimers and longer strings of adatoms sitting in the troughs. The energies obtained from DFT-PW91 could easily be in error by $0.1 \mathrm{eV}$, but we have also neglected entropic effects, both in the relative population of $S$ and $P$ sites and in the transition state theory estimate of the rates, where entropic effects and recrossing corrections can easily cause the prefactor for different paths to differ by a couple of orders of magnitude. Treating the prefactor for the edge diffusion hops as an adjustable parameter, we re-ran the $\mathrm{kMC}$ simulations until roughly $30 \%$ of the dimers form in the troughs at room temperature. Then some $S \cdots S-S$ trimers also form. The prefactor needed to be increased by a factor of 5 to achieve this $[22,23]$.

Eqs. (1) and (2) then predict a crossover in the relative importance of the two diffusion paths near $300 \mathrm{~K}$. This is clearly reflected in $\mathrm{kMC}$ simulations of deposition onto a $400 \mathrm{~K}$ substrate. Now $80 \%$ of the deposited atoms end up as dimers or longer $S$ strings (some four atoms long) in the troughs, and only $20 \%$ end up as $P-P$ dimers. Because of the metastability of the $S-S$ dimers and longer $S$ strings, their abundance is very sensitive to deposition rate and the overall concentration of adatoms. In particular, an adatom diffusing "on top" into a neighboring $P$ site can take one of the end atoms of an $S$ string to form a $P-P$ dimer. If the initial feature is only a single $S-S$ dimer, this leaves a new adatom free to join or disrupt other $S-S$ dimers in the system. Also, an adatom coming into a $P$ site adjacent to a three atom $S$ string will most likely disrupt the $S$ string to form a $P-P$ on-top dimer and an $S-S$ dimer (this is downhill in energy by $0.5 \mathrm{eV}$ ). The survival of $S$ dimers and $S$ strings, therefore, hinges on the fact that there is a shift from on-top diffusion to edge diffusion in our model. If initially there is a longer $S$ string it is likely to stay in place and further additional atoms will produce strings of $P-P$ dimers. This allows $S$ strings to develop into regular dimer rows at higher coverage.

The large abundance of $S-S$ dimers and longer $S$ strings at $400 \mathrm{~K}$ is consistent with the results of experiments by Bedrossian and by Zhang et al. There, samples prepared by room temperature deposition were annealed 
at $400 \mathrm{~K}$ and then quenched for imaging. Bedrossian found that $S$ strings became the prevalent adsorbate configurations [12]. Zhang et al. reported the fraction of dimers on top decreased from 0.7 to 0.5 in $12 \mathrm{sec}$ of annealing at $400 \mathrm{~K}$ [5].

This experimental result was interpreted in terms of direct addimer diffusion and used to infer that dimers are more stable in the troughs [5], in clear contradiction with DFT calculations. But Swartzentruber [13] has recently shown that this mechanism is not effective on clean, defect free parts of the surface. We have yet to identify a mechanism by which on-top addimers would disappear during annealing (our kMC calculations simulated deposition). The rate reported by Zhang et al. [5] suggests an activation barrier of $1.2 \mathrm{eV}$, close to the estimated $1.3 \mathrm{eV}$ activation barrier for removing adatoms from steps [23]. Thus this barrier could correspond to direct dissociation of the dimers, but it is also possible that the low energy path requires defect sites or the participation of other adatoms on the surface.

To summarize, an adatom diffusion model with two primary paths for diffusion parallel to dimer rows, based on DFT-PW91 calculations of adatom and addimer energetics, can explain puzzling features observed in recent STM experiments. At low temperatures adatoms hop along the tops of dimer rows, resulting in the formation of on-top addimers. At $160 \mathrm{~K}$ this occurs only for atoms landing on top of dimer rows. At $300 \mathrm{~K}$ all the adatoms form addimers or larger structures, again mostly on top because the ontop diffusion path continues to dominate. After slight adjustment of the edge diffusion prefactor, about $30 \%$ of the deposited atoms end up in the trough in agreement with experiment. Deposition at $400 \mathrm{~K}$ then mainly leads to formation of dimers and strings of adatoms sitting in the trough as the edge diffusion path becomes dominant. The annealing experiments of Bedrossian and of Zhang et al. can then be reconciled with the DFT-PW91 calculations, if the on-top addimers that form at room temperature dissociate in the $400 \mathrm{~K}$ annealing process. Our kMC simulations indicate that the on-top dimers would not reform at $400 \mathrm{~K}$ because of the shift in adatom diffusion mechanism. Our calculations illustrate two important features which seem to apply to the $\mathrm{Si}(100)$ system and could well apply to other systems: (1) The diffusion path with the lowest overall activation energy barrier is not necessarily the dominant diffusion mechanism, and (2) the metastable structure formed at a given temperature is not necessarily thermodynamically more stable than a metastable structure formed at a lower temperature, a consequence of kinetic effects in a system far from equilibrium.

We gratefully acknowledge communications with Peter Bedrossian, Brian Swartzentruber, Bob Wolkow, and Z.-Y. Zhang. H. J. would like to thank CAMP (Center for Atomic-scale Materials Physics) and the Physics Department of the Technical University of Denmark for hospitality and support, and Horia Metiu for several stimulating discussions. This work was supported by the National Science Foundation under award No. CHE9217294 (CARM). Computer time was made available on a Paragon parallel supercomputer by the San Diego Supercomputer Center.

[1] E. Kaxiras, Computational Materials Science (to be published).

[2] G. Brocks, P. J. Kelly, and R. Car, Phys. Rev. Lett. 66, 1729 (1991); Surf. Sci. 269/270, 860 (1992).

[3] R. A. Wolkow, Phys. Rev. Lett. 74, 4448 (1995).

[4] Y.-W. Mo, J. Kleiner, M. B. Webb, and M. G. Lagally, Surf. Sci. 268, 275 (1991); Phys. Rev. Lett. 66, 1998 (1991).

[5] Z.-Y. Zhang, F. Wu, H. J. W. Zandvliet, B. Poelsema, H. Metiu, and M. G. Lagally, Phys. Rev. Lett. 74, 3644 (1995).

[6] J. P. Perdew and Y. Wang (unpublished); J. P. Perdew, in Electronic Structure of Solids '91, edited by P. Ziesche and H. Eschrig (Akademie Verlag, Berlin, 1991).

[7] A. P. Smith, J. Wiggs, H. Jónsson, H. Yan, L. R. Corrales, P. Nachtigall, and K. Jordan, J. Chem. Phys. 102, 1044 (1995).

[8] J. K. Wiggs and H. Jónsson, Comp. Phys. Commun. 81, 1 (1994); 87, 319 (1995).

[9] R. Car and M. Parrinello, Phys. Rev. Lett. 55, 2471 (1985).

[10] G. Brocks and P. J. Kelly, Phys. Rev. Lett. 76, 2362 (1996).

[11] T. Yamasaki, T. Uda, and K. Terakura, Phys. Rev. Lett. 76, 2949 (1996).

[12] P. J. Bedrossian, Phys. Rev. Lett. 74, 3648 (1995).

[13] B. S. Swartzentruber, Phys. Rev. Lett. 76, 459 (1996).

[14] Z.-Y. Zhang and H. Metiu, Surf. Sci. 248, L250 (1991).

[15] However, the incident $\mathrm{Si}$ atom is in a triplet state and could be reacting to a very different potential energy surface than the one describing the interaction of an adsorbed Siatom with the substrate.

[16] The DFT-LDA calculations give equally high barriers for $P \rightarrow P$ hops as for $P \rightarrow S$ hops.

[17] Y.-T. Lu, Z. Zhang, and H. Metiu, Surf. Sci. 257, 199 (1991).

[18] F. H. Stillinger and T. A. Weber, Phys. Rev. B 31, 5262 (1985).

[19] The SW potential predicts the adatom breaks the underlying dimer as it diffuses along a dimer row, while in the DFT results the underlying dimer is only slightly stretched. Also, the SW potential favors an epitaxial site midway between rows rather than the $S$ site for binding the single adatom.

[20] Y.-W. Mo, R. Kariotis, B. S. Swartzentruber, M. B. Webb, and M. G. Lagally, J. Vac. Sci. Technol. A 8, 201 (1990).

[21] A. F. Voter, Phys. Rev. B 34, 6819 (1986).

[22] Such small variation in a prefactor cannot, at present, be predicted from DFT calculations. See P.E. Blöchl, C. G. Van de Walle, and S. T. Pantelides, Phys. Rev. Lett. 64, 1401 (1990).A larger prefactor $\nu_{S \rightarrow S}$ is consistent with the so-called "compensation rule." See G. Boisvert, L. J. Lewis, and A. Yelon, Phys. Rev. Lett. 75, 469 (1995).

[23] B. S. Swartzentruber and M. Schacht, Surf. Sci. 322, 83 (1995). 